\title{
Oh Heavy Burden: Recognizing the Risk of Venous Thromboembolism in Women Undergoing Assisted Reproduction
}

\author{
Gregory Piazza ${ }^{1}$ \\ ${ }^{1}$ Division of Cardiovascular Medicine, Department of Medicine, \\ Brigham and Women's Hospital, Harvard Medical School, Harvard \\ University, Boston, Massachusetts, United States \\ Thromb Haemost 2018;118:2011-2013.
}

"With what price we pay for the glory of motherhood."

- Isadora Duncan

Data from the United States Center for Disease Control and Prevention (CDC) show that a growing number of women are undergoing assisted reproductive technology (ART) cycles, including in vitro fertilization (IVF), each year. ${ }^{1}$ The European Society of Human Reproduction and Embryology has reported a similar trend. ${ }^{2}$ As our understanding of the pathophysiology of infertility deepens and indications for ART broaden, the proportion of couples bearing the financial and psychological cost of infertility treatments can be expected to increase. The risk of thrombosis, in particular venous thromboembolism (VTE), represents a critical additional burden of ART. Unlike the risk of VTE associated with hormonal contraception, pregnancy and the post-partum period, the danger of thrombotic complications of ART is often under-appreciated by patients and under-recognized by providers. Accordingly, women undergoing ART may be left to bear this burden alone.

The pathogenesis of VTE in ART is believed to rely upon a combination of underlying patient-specific risk factors and consequences of the prescribed treatments and resultant pregnancies (-Fig. 1). Traditional VTE risk factors such as obesity, smoking, thrombophilia and inflammatory disorders frequently co-exist with and may contribute to infertility. For example, in a study of 559 pregnant patients with and 1,229 without VTE, traditional risk factors of high body mass index and limited mobility, in addition to conception via ART and multiple gestations, increased the risk of antepartum VTE. ${ }^{3}$

ART, specifically IVF, results in a high oestrogen state that favours thrombosis through several suspected mechanisms, including an increase in pro-coagulant factors, acquired

\begin{abstract}
Address for correspondence Gregory Piazza, MD, MS, Division of Cardiovascular Medicine, Brigham and Women's Hospital, 75 Francis Street, Boston, MA 02115, United States (e-mail: gpiazza@bwh.harvard.edu).
\end{abstract}

activated protein $\mathrm{C}$ resistance, down-regulated fibrinolysis and relative deficiencies of endogenous anticoagulants, such as protein $\mathrm{S}{ }^{4}$ The increased risk of VTE associated with ART ranges between three- and fourfold across cohort studies compared with pregnancies resulting from natural conception. $^{5,6}$ Multiple gestations appear to augment the risk while early pregnancy losses are associated with a low absolute risk of VTE following ART. ${ }^{5,7}$ A sub-set of women who develop ovarian hyper-stimulation following ovulation induction bear a considerable risk of venous and arterial thrombosis. ${ }^{8}$

In the previous issue of Thrombosis and Haemostasis, Grandone et al provided additional perspective on the risk of VTE in women undergoing ART using data from the prolific RIETE Registry. ${ }^{9}$ In brief, the investigators analysed this large multicentre registry to assess thrombosis risk factors and the frequency of ART-associated VTE. Of 6,718 women with VTE, 41 suffered an event related to ART. Among 22 of these women who underwent a hyper-coagulable work-up, half were diagnosed with a thrombophilia, suggesting at least $27 \%$ prevalence. Consistent with other analyses, ovarian stimulation was associated with an increased risk of VTE, especially during the first trimester. Unexpectedly, the risk of pulmonary embolism (PE) was fourfold higher with unsuccessful ART cycles compared with successful ones resulting in pregnancy. This observation contrasts an earlier study by Grandone et al that demonstrated that VTE was less frequent following failed ART cycles compared with the successful ones. ${ }^{10}$

The current analysis not only raises awareness of the overall burden of VTE in patients undergoing ART, but also highlights the potential danger of PE following failed cycles. The CDC's 2015 Fertility Clinic Success Rates Report demonstrated that 231,936 ART cycles resulted in 74,665 pregnancies and 157,271 failed cycles. ${ }^{1}$ These data suggest a large received

October 31, 2018

accepted

October 31, 2018 (c) 2018 Georg Thieme Verlag KG Stuttgart · New York
DOI https://doi.org/ 10.1055/s-0038-1676073. ISSN 0340-6245. 


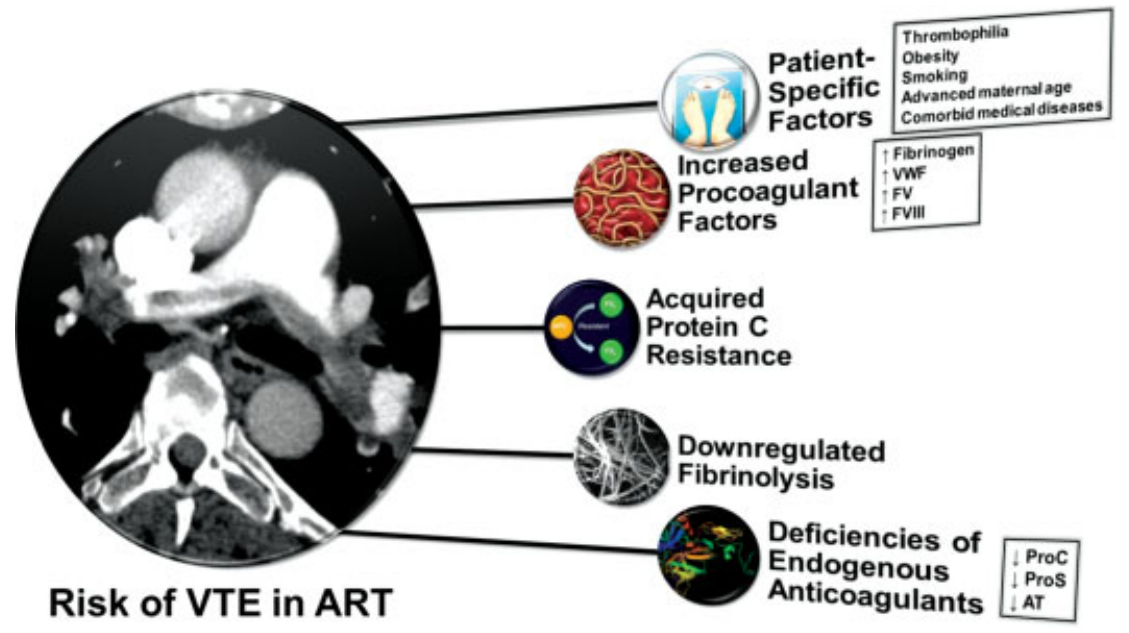

Fig. 1 Proposed pathogenetic mechanisms for venous thromboembolism (VTE) in assisted reproductive technology (ART). AT, anti-thrombin; FV, factor V; FVIII, factor VIII; ProC, protein C; ProS, protein S; VWF, von Willebrand factor.

potential population at risk for VTE, especially considering that women who suffer failed cycles commonly undergo multiple subsequent cycles with repeated exposure to hormonal therapies, including ovarian stimulation. Advanced maternal age and co-morbid medical illnesses, both commonly observed in women who have failed cycles, further amplify the VTE risk. Not to be under-estimated, concomitant physical and psychological stress from failed cycles and recurrent miscarriage may contribute to immobility and other lifestyle factors that further drive thrombosis risk.

Grandone et al deserve recognition for adding to our understanding of the relationship between ART and thrombosis. The RIETE Registry is a rich data repository that captures a patient population representative of that encountered in clinical practice and that has provided valuable insights into many aspects of thrombosis. The granularity of the data presented here, including patient characteristics, thrombophilia testing, details of the ART regimens and cycle outcomes, is a critical strength of the analysis. The limitation of the small sample size of women suffering VTE in the setting of ART in this cohort can be overcome through subsequent data collections.

Hopefully, this article by Grandone et al will serve as a catalyst for further studies focused on the association of thrombosis and infertility, including ART. A multi-disciplinary, collaborative approach between thrombosis specialists and infertility experts is most likely to yield the greatest success. We recently launched the Fertility, Hypercoagulability, and Inflammation (FREYA) Registry, named for the Norse goddess of fertility, to merge the expertise of our Brigham and Women's Hospital Thrombosis Research Group and Center for Infertility and Reproductive Surgery and to better understand the burden of thrombosis in our ART patients. Further investigation, ranging from registry science to mechanistic studies, into this crossroads of thrombosis and infertility will provide us with tools to identify women at increased risk for VTE in the setting of ART who may benefit from thrombophilia testing, adjustments to IVF regimens aimed at mitigating thrombotic risk and prescription of thromboprophylaxis. Unfortunately, current evidence-based guidelines to protect women undergoing ART from thrombotic complications are limited by a dearth of data. ${ }^{11,12}$

As mechanisms for infertility continue to be elucidated and options for ART expand, an increasing number of infertile couples will be given the hope of parenthood. In addition to financial considerations, the physical and psychological price of infertility treatment will be considerable, and often, disproportionately paid by women. Research such as the current analysis aimed at understanding the risk of VTE with ART and eventually defining pathways for mitigating this danger is critical to ensure women who suffer from infertility are not left to carry this heavy burden alone.

\section{Conflict of Interest}

Dr. Piazza reports grants from BMS/Pfizer, Janssen, DaiichiSankyo, Bayer and Portola, outside the submitted work.

\section{References}

1 Centers for Disease Control and Prevention, National Center for Chronic Disease Prevention and Health Promotion. Division of Reproductive Health. Available at: http://nccd.cdc.gov/drh_art. Accessed October 22, 2018

2 Calhaz-Jorge C, de Geyter C, Kupka MS, et al; European IVFMonitoring Consortium (EIM) for the European Society of Human Reproduction and Embryology (ESHRE). Assisted reproductive technology in Europe, 2012: results generated from European registers by ESHRE. Hum Reprod 2016;31(08):1638-1652

3 Jacobsen AF, Skjeldestad FE, Sandset PM. Ante- and postnatal risk factors of venous thrombosis: a hospital-based case-control study. J Thromb Haemost 2008;6(06):905-912

4 Gurunath S, Vinekar S, Biliangady R. Assisted reproductive techniques in a patient with history of venous thromboembolism: a case report and review of literature. J Hum Reprod Sci 2018;11(02):193-197

5 Hansen AT, Kesmodel US, Juul S, Hvas AM. Increased venous thrombosis incidence in pregnancies after in vitro fertilization. Hum Reprod 2014;29(03):611-617

6 Villani M, Dentali F, Colaizzo D, et al. Pregnancy-related venous thrombosis: comparison between spontaneous and ART conception in an Italian cohort. BMJ Open 2015;5(10):e008213 
7 Hansen AT, Juul S, Knudsen UB, Hvas AM. Low risk of venous thromboembolism following early pregnancy loss in pregnancies conceived by IVF. Hum Reprod 2018;33(10):1968-1972

8 Chan WS. The 'ART' of thrombosis: a review of arterial and venous thrombosis in assisted reproductive technology. Curr Opin Obstet Gynecol 2009;21(03):207-218

9 Grandone E, Di Micco PP, Villani M, et al; RIETE Investigators. Venous thromboembolism in women undergoing assisted reproductive technologies: data from the RIETE Registry. Thromb Haemost 2018;118(11):1962-1968

10 Villani M, Favuzzi G, Totaro P, et al. Venous thromboembolism in assisted reproductive technologies: comparison between unsuc- cessful versus successful cycles in an Italian cohort. J Thromb Thrombolysis 2018;45(02):234-239

11 Bates SM, Greer IA, Middeldorp S, Veenstra DL, Prabulos AM, Vandvik PO. VTE, thrombophilia, antithrombotic therapy, and pregnancy: Antithrombotic Therapy and Prevention of Thrombosis, 9th ed: American College of Chest Physicians EvidenceBased Clinical Practice Guidelines. Chest 2012;1412 (Suppl): e691S-e736S

12 Bates SM, Middeldorp S, Rodger M, James AH, Greer I. Guidance for the treatment and prevention of obstetric-associated venous thromboembolism. J Thromb Thrombolysis 2016;41 (01):92-128 